DOI: https://doi.org/10.14311/TPFM.2022.011

\title{
EXPERIMENTAL VALIDATION OF GRANULAR FLOW KINETIC THEORY UNDER TURBULENT FLOW CONDITIONS
}

\author{
J. Haidl, Z. Chára, V. Matoušek
}

The Czech Academy of Sciences, Institute of Hydrodynamics, Pod Paťankou 30/5, 16612 Prague 6, Czech Republic.

\begin{abstract}
The mixed classical and extended kinetic theory of granular flows is used for modeling the characteristics of particles-water turbulent sheet flow. The open-source solver sedFoam v3.1 is used for the 1-D and 2-D flow simulations. The simulation results are compared to the experimental data measured in the open channel. After that, the simulation parameters are optimized to achieve the best possible agreement between the simulation and the experimental results. The unsatisfactory performance of the KT models and the observed simulation instabilities are discussed.
\end{abstract}

Keywords: Kinetic theory, granular flow, sedFoam.

\section{Introduction}

The turbulent granular flows occur in a multitude of natural and industrial processes. In nature, the sediment transport during the floods was and remains the key factor responsible for the fertile soil redistribution, whilst the capability of the gas or liquid flows to carry the solid particles is leveraged for example in the transport of fine-grained ore or granular products of the polymer industry. Thanks to their widespread and importance, it is desirable to develop a reliable and computationally realizable method of granular flow modeling.

Mathematically, there are two main approaches to the granular flow modeling: (1) EulerianLagrangian approach, and (2) Eulerian-Eulerian aproach. In the first approach, the equations of the movement are solved for each individual particle, limiting the problem size to the computationally realizable domain resolution and particle count. The latter approach treats both the fluid and the particles as a continuum - physical and hypothetical phases. This enables solving the transport equations for both phases avoiding the limitations for domain resolution and particles count.

In this work, we examine the Eulerian-Eulerian approach to the granular flow modeling in highly turbulent flow conditions. The physical properties of the hypothetical fluid of particles are calculated using the Kinetic Theory of granular flows and the resulting set of differential and algebraic equations is solved by means of the sedFoam v3.1 solver [1], built on the open-source OpenFOAM library [2].

The solver is altered to use the recent formulations of the kinetic theory and a series of 1-D and 2-D simulations is performed. The solver ability to predict the experimental data measured at The Czech Technical University is tested from the point of reliability and computational expenses.

\section{Problem formulation}

The Eulerian formulation of the granular flows and its actual implementation in the CFD solver sedFoam v3.1 [1] used in this work is described in detail by Chauchat et al. [3] and the literature referenced therein. Therefore, we provide only a brief overview of the problem and the necessary references.

Let's assume the two-phase flow problem with a hypothetical fluid phase $a$ representing the suspended particles and a liquid phase $b$ representing the carrier liquid [4]. Mathematically, the presented problem comprises the transport equations for both fluid phases $(k=a, b)$

$$
\frac{\partial \rho^{k} \alpha^{k} u_{i}^{k}}{\partial t}+\frac{\partial \rho^{k} \alpha^{k} u_{i}^{k} u_{j}^{k}}{\partial x_{j}}=-\alpha^{k} \frac{\partial p}{\partial x_{i}}+\frac{\partial \tau_{i j}^{k}}{\partial x_{j}}+\alpha^{k} \rho^{k} g_{i} \pm D_{i}
$$


together with the mass conservation equations for both phases

$$
\frac{\partial \alpha^{k}}{\partial t}+\frac{\partial \rho^{k} \alpha^{k} u_{i}^{k}}{\partial x_{i}}=0
$$

In this work, the problem was solved utilizing the URANS approach with turbulent quantities modeled by means of the $k-\epsilon$ model modified for granular flows [5]. The turbulence model employs two empirical coefficients, turbulent Schmidt number $\boldsymbol{\sigma}_{\mathbf{c}}$ expressing the ratio of turbulent viscosity to particle diffusivity and the drag-induced damping coefficient $\boldsymbol{B}$, describing the dependence of liquid turbulence suppression on the particle concentration. These two quantities represent the adjustable parameters of the model.

The term $D_{i}$ in the Eq. (1) represents the interaction of the fluid and the particles. It should be added to the momentum equation for the particles and subtracted from the equation for the fluid.

$$
D_{i}=\alpha^{a} \alpha^{b} K\left(u_{i}^{b}-u_{i}^{a}\right)-\frac{1}{\sigma_{\mathrm{c}}} \alpha^{b} K \nu_{\mathrm{t}} \frac{\partial \alpha^{a}}{\partial x_{i}}
$$

For the liquid phase $(b)$, the divergence of the stress tensor $\tau_{i j}^{b}$ is calculated from the liquid bulk and turbulent viscosity and velocity Laplacian

$$
\frac{\partial \tau_{i j}^{b}}{\partial x_{j}}=\left(\mu^{b}+\mu_{\mathrm{t}}\right) \frac{\partial^{2} u_{j}^{b}}{\partial x_{i}^{2}}
$$

while for the solid phase $(a)$ the stress $\tau_{i j}^{a}$, resulting from particle-particle interactions, is modeled using the kinetic theory (KT) of granular mixtures (for example [4, 3]).

$$
\tau_{i j}^{a}=2\left(\mu^{a}+\mu_{f}^{a}\right) S_{i j}^{a}+\left[\xi^{a} \frac{\partial u_{k}^{a}}{\partial x_{k}}-\left(\tilde{p}_{\mathrm{c}}^{a}+\tilde{p}_{f}^{a}\right)\right] \delta_{i j} \quad S_{i j}^{a}=\frac{1}{2}\left(\frac{\partial u_{i}^{a}}{\partial x_{j}}+\frac{\partial u_{j}^{a}}{\partial x_{i}}\right)-\frac{1}{3} \frac{\partial u_{k}^{a}}{\partial x_{k}} \delta_{i j}
$$

The KT modeling is based on tracking of the fluctuating particle velocity $u_{i}^{\prime}$ by means of the granular temperature $\Theta=\left(u_{i}^{\prime}\right)^{2}$.

$$
\frac{3}{2}\left[\frac{\partial \rho^{a} \alpha^{a} \Theta}{\partial t}+\frac{\partial \rho^{a} \alpha^{a} u_{j}^{a} \Theta}{\partial x_{j}}\right]=\left(\tilde{p}_{f}^{a} \delta_{i j}+\tau_{i j}^{a}\right) \frac{\partial u_{i}^{a}}{\partial x_{j}}+\frac{\partial}{\partial x_{j}}\left(\lambda^{a} \frac{\partial \Theta}{\partial x_{j}}\right)-\gamma+S_{\mathrm{t}}
$$

Analogously to the kinetic theory of gases, the granular flow collision pressure $\left(\tilde{p}_{c}^{a}\right)$ and transport properties (shear viscosity $\mu^{a}$, bulk viscosity $\xi^{a}$, granular kinetic energy conductivity $\lambda^{a}$ can be calculated. The fluctuating kinetic energy of particles is transported with the flow of the particles and via the energy conductivity $\lambda^{a}$, generated by the particle shear stress $\tau^{a}$ and by means of the liquid turbulence (the term $S_{\mathrm{t}}$ ), and dissipated via the inelastic collisions (the term $\gamma$ ).

In this work, we use a blend of two formulations of the KT according to the particle concentration $\alpha^{a}$. For the collisional regime $\left(\alpha^{a}<0.49\right.$, subscript $\left.\mathrm{C}\right)$, the classical KT relations, derived by Garzó and Dufty [6], are used, while for the dense granular flow (subscript E), we use the extended KT formulated by Berzi and Vescovi [7]. To suppress the steep change of the calculated properties at $\alpha^{a}=0.49$, the sigmoidal transition function $t$ is used. The individual terms used in this work are summarized in Tab. 1.

\section{$3 \quad$ Experimental data}

To validate the simulation results, we used the experimental data measured with the water and plastic granular particles (code name SUN25) in the open channel described in [8]. The open channel is $8 \mathrm{~m}$ long, $0.2 \mathrm{~m}$ wide with the maximal depth of $0.25 \mathrm{~m}$. The properties of the particles are listed in Tab. 2. During the experiments, the water and the particles circulated in a closedloop, the data were recorded after the steady-state characterized by constant phase flow rates was reached.

The experimental results for the internal structure of the flow was collected using camera-based methods in the laboratory flume. A stereoscopic particle tracking algorithm has been used to evaluate the velocity profiles; a laser-stripe technique has been employed to measure the concentration profiles. 
Table 1: The KT relations used in this work. Quantities denoted by a subscript C follow the classical KT [6, 3], whilst the subscript E indicates the extended KT [7]. If omitted, a superscript $a$ is tacitly assumed, i.e., $\alpha=\alpha^{a}, \rho=\rho^{a}$, etc.

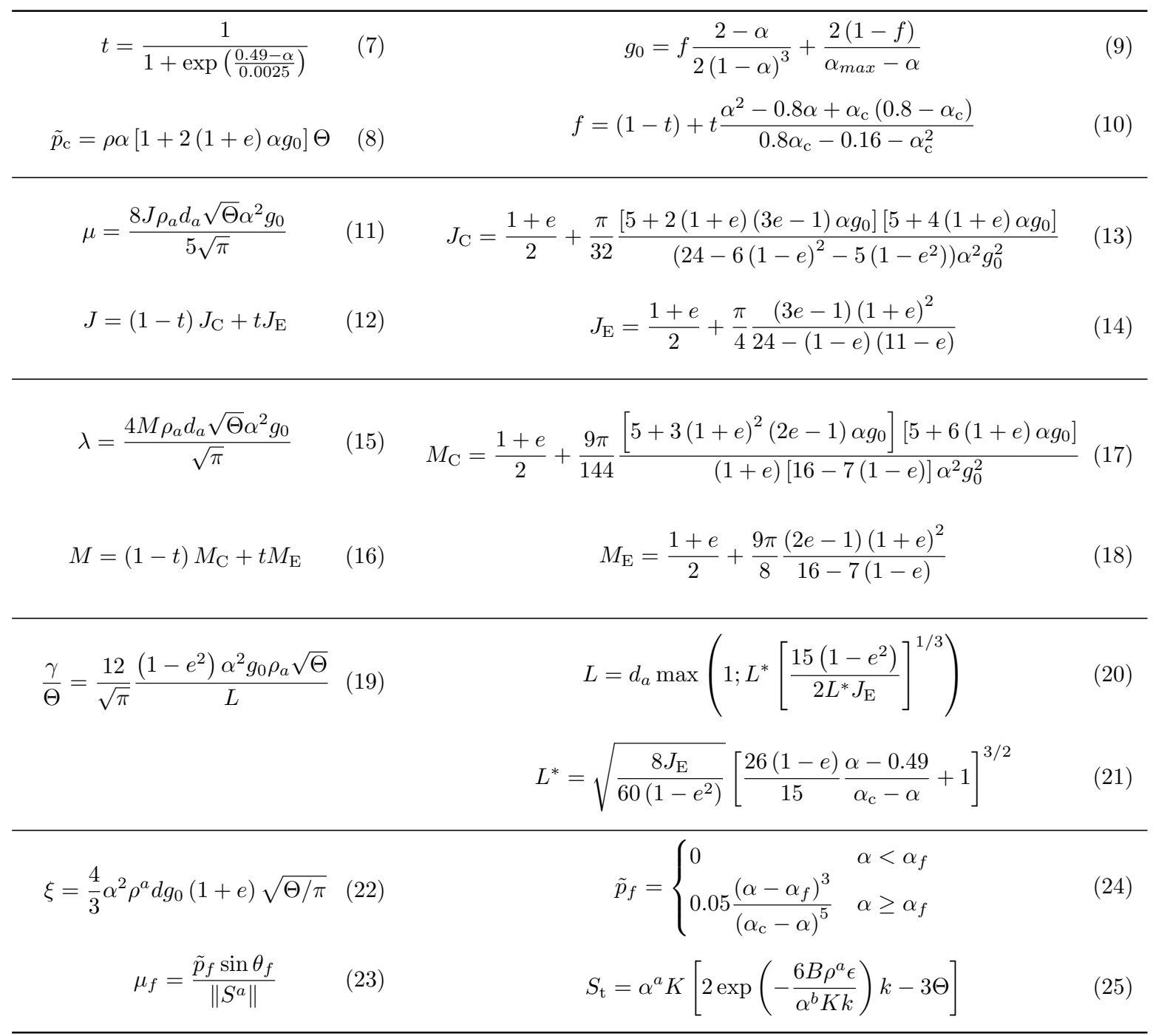

For the laser-stripe method, two sources of uncertainty are evaluated to estimate the measurement error. First, the systematic error caused by the method itself is estimated as a standard deviation between known concentrations adjusted in calibration experiments and concentrations evaluated from these experiments after the method is calibrated. Its value from the calibration experiments for SUN25 is 0.024 . Second, the random error for measurement of local concentration is estimated. A root mean square average of the standard deviations calculated from all positions in SUN25 concentration profiles is 0.025 . The combined total measurement error value is 0.034 .

For the particle tracking method, the key source of uncertainty is an identification of particles in a frame and their matching to particles in the next frame. For typical granular flows, the percentage of successful matches is near 90\% [9] and the resulting error confidence band of measured local velocity fluctuations, expressed in terms of the local granular temperature, is of about $\pm 3.5 \%$ [8]. This information complies with a very good agreement between the local velocities inside the collisional transport layer measured by the cameras and by the ultrasonic velocity profiler [8].

Three data sets, characterized by the phase flow rates $\left(Q^{a}, Q^{b}\right)$, concentration and velocity profiles along the channel height, $\alpha^{a}$ and $U$, respectively, were used as the basis for the simulations. The characteristic values of each data set are listed in Tab. 3. 
Table 4: Boundary conditions used in this work. If not specified, a zero gradient BC is used.

\begin{tabular}{lcc}
\hline patch & 1-D simulation & 2-D simulation \\
\hline bottom (wall) & $U^{k}:$ no slip $; k, \epsilon$ : wall functions \\
top (free surface) & \multicolumn{2}{c}{$U^{k}:$ slip $; p: 0$} \\
left (inlet) & all: cyclic & $U^{k}:\left(U_{x, \text { in }}^{k}, 0,0\right) ; \alpha^{a}: \alpha_{\text {in }}^{a}$ \\
right ( outlet) & all: cyclic & $U^{k}:$ inlet $/$ outlet \\
front & & all: empty \\
back & & \\
\hline
\end{tabular}

Table 2: Physical properties of the water-particles suspension.

\begin{tabular}{lrl}
\hline$d_{\mathrm{eq}}$ & 2.8 & $\mathrm{~mm}$ \\
$\rho^{a}$ & 1280 & $\mathrm{~kg} \cdot \mathrm{m}^{-3}$ \\
$e$ & 0.80 & \\
$\varphi^{a}$ & 0.565 & \\
$\rho^{b}$ & 997 & $\mathrm{~kg} \cdot \mathrm{m}^{-3}$ \\
$\mu^{b}$ & 0.89 & $\mathrm{mPa} \cdot \mathrm{s}$ \\
\hline
\end{tabular}

Table 3: Overview of experimental data sets and the corresponding inlet boundary conditions.

\begin{tabular}{lcccc}
\hline ID & $\begin{array}{c}Q^{a} \\
\left(\mathrm{dm}^{3} \cdot \mathrm{s}^{-1}\right)\end{array}$ & $\begin{array}{c}Q^{b} \\
\left(\mathrm{dm}^{3} \cdot \mathrm{s}^{-1}\right)\end{array}$ & $\begin{array}{c}U_{x, \text { in }}^{k} \\
(-)\end{array}$ & $\begin{array}{c}\alpha_{\text {in }}^{a} \\
(-)\end{array}$ \\
\hline T7 & 5.10 & 0.902 & 0.300 & 0.150 \\
T10 & 5.44 & 0.752 & 0.310 & 0.122 \\
T19 & 10.0 & 0.214 & 0.511 & 0.0209 \\
\hline
\end{tabular}

\section{Methodology}

We explore the ability of the kinetic theory of granular flows to predict the concentration and velocity profiles measured using the slightly tilted flume (inclination angle bellow $2^{\circ}$ ). $1-\mathrm{D}$ and 2-D simulations are performed with the sedFoam v3.1 solver [1] using the customized relations for the kinetic theory quantities calculation (see Tab. 1). For the lack of computational power, the 3-D simulations were not performed.

The computational domain used in this work corresponds to the rectangular channel $5 \mathrm{~m}$ long ( $x$-direction), $0.1 \mathrm{~m}$ deep ( $y$-direction) and $0.2 \mathrm{~m}$ wide ( $z$-direction). The simulations are performed on an orthogonal grid of multiple refinements. In the case of 1-D simulations, the channel height is divided to $\{50,100,200\}$ cells, for the case of $2-\mathrm{D}$ simulations, the refinements of $\{2500 \times 100,5000 \times 200\}$ cells. The boundary conditions setting the $1-\mathrm{D}$ and $2-\mathrm{D}$ cases are listed in Tab. 4.

In the case of 1-D simulation, the boundary conditions do not allow to prescribe the phase flow rates corresponding to the experimental conditions, therefore an alternative procedure was developed to match the simulated and experimental conditions. The phase velocities are adjusted by means of an additional source term in Eq. (1) with the meaning of an arbitrary pressure gradient in the $x$-direction, $\partial p_{\mathrm{A}} / \partial x$, and the solid phase flow rate is adjusted via the initial concentration of the particles in the domain, $\alpha_{a}^{0}$. To find the values of these two parameters, the Python-based framework was designed, implementing either the Wolfe's method for the non-linear equation systems or the Nelder-Mead algorithm for the non-linear optimization -see the next section. In every iteration of the algorithm, corresponding IC's and BC's are set and the simulation runs until a stationary solution is attained.

In the case of 2-D simulation, the experimental phase inlet velocities and particle fraction, $U_{x, \text { in }}^{k}$ and $\alpha_{\text {in }}^{a}$, respectively, are set directly and the simulation starts with initial conditions $U^{k}=(0,0,0)$, $\alpha^{a}=0$. During the simulation, the channel is filled with particles, which form a stationary bed at the bottom of the channel, thus reducing its open cross-section and increasing the flow velocity. This process continues until the water flow velocity, capable to carry the inflow of the particles, is attained. The liquid and particle flow rate at the channel outlet is monitored indicating the steady-state of the simulation. The steady-state concentration and velocity profiles are sampled at the coordinate $x=4.00 \mathrm{~m}$ from the channel inlet, eliminating the influence of the inlet and the outlet BCs. This approach was validated by comparison of the profiles sampled at the positions 
$0.5 \mathrm{~m}$ in front and behind the sampling coordinate. All three samples provided the profiles in an acceptable agreement.

\section{Results and discussion}

\section{$5.1 \quad 1-\mathrm{D}$ simulations}
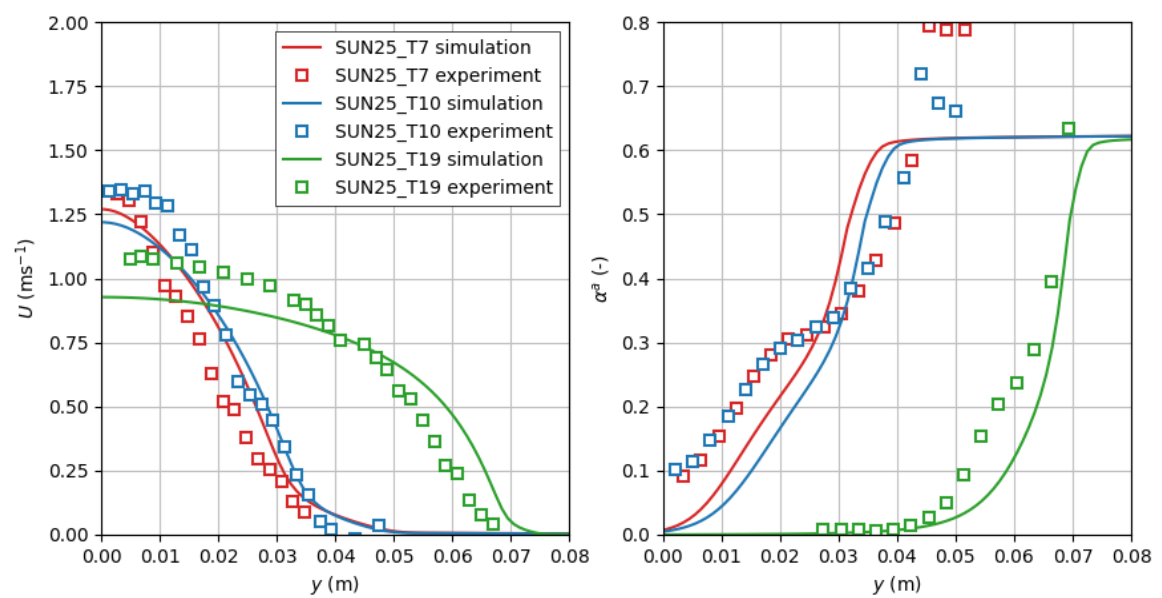

Figure 1: Velocity and particle concentration profiles calculated with the 1-D simulation and original values of $\sigma_{\mathrm{c}}=0.449$ and $B=0.25$.

For the 1-D simulations, there are five parameters outside the KT, which can be set or adjusted to obtain the best possible agreement between the simulation and experimental results. There are two parameters, $\left(\nabla p_{\mathrm{A}}\right)_{x}=\partial p_{\mathrm{A}} / \partial x$ and $\alpha_{0}^{a}$, which determine the phase flow rates; parameters $\sigma_{\mathrm{c}}$ and $B$, related to the interaction of particles and liquid turbulence; and the shape factor $\varphi^{a}$, which adjusts the liquid-particle drag force.

First, we tested the agreement of KT simulation with the experimental velocity and concentration profiles. The values of $\sigma_{\mathrm{c}}=2.27$ and $B=0.25$, recommended in the [3], based on results of [10]. The value of the shape factor $\varphi$, determined from the particle settling velocity, was used. The comparison of the simulated and the experimental profiles for the cases is presented in Fig. 1.

It is evident, that the recommended values do not provide an acceptable agreement between the experiment and the simulation. Neither the height of the stationary bed nor the shapes of the concentration and velocity profiles are predicted correctly. This outcome suggests: (i) the values of parameters $B$ and $\sigma_{\mathrm{c}}$, valid for the test cases of $[3,10]$, must be adjusted for each material individually; (ii) the 1-D simulation is not capable to evaluate sufficiently the fluidparticle interactions via the turbulent quantities; (iii) the KT relations are not tuned properly to the particles used in this work.

In order to test the first hypothesis, the optimization of the parameters $\left\{\left(\nabla p_{\mathrm{A}}\right)_{x}, \alpha_{0}^{a}, B, \sigma_{\mathrm{c}}, \varphi^{a}\right\}$ was performed using the Nelder-Mead method in order to obtain: 1) the best agreement of $\alpha(y)$ values, and 2) the best agreement of $\alpha(y)$ and $U_{x}(y)$ values. The results of the optimization for both cases are shown in Figs. 2 and 3.

The presented results show clearly, that with the 1-D simulation of the flow problem and using the KT relations summarized in Tab. 1, the acceptable agreement between the experimental and calculated concentration profiles can be achieved only with significantly different phase flow rates than measured experimentally. The optimization with respect to the agreement of both, the concentration and the velocity profiles yield considerably worse results.

In addition, the optimization in all three tested cases (T7, T10 and T19) provides the value of $B$ close to zero. According to the physical meaning of this parameter, the results suggest that there is no significant correlation between the fluid and particle velocity fluctuations. 

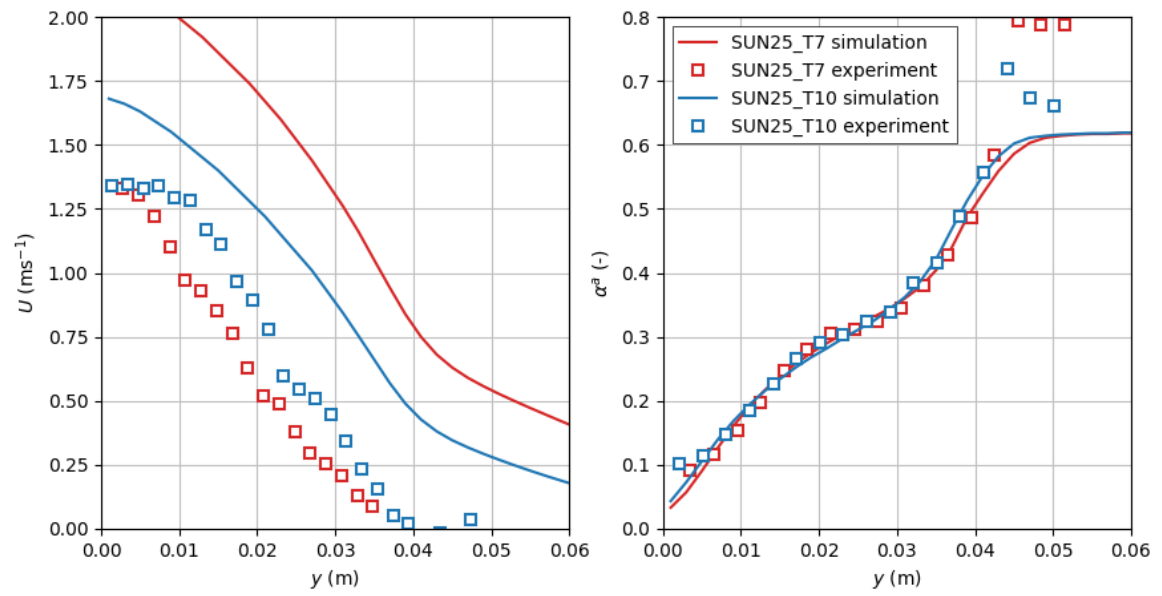

Figure 2: Velocity and particle concentration profiles optimized for the best agreement in $\alpha^{a}$.
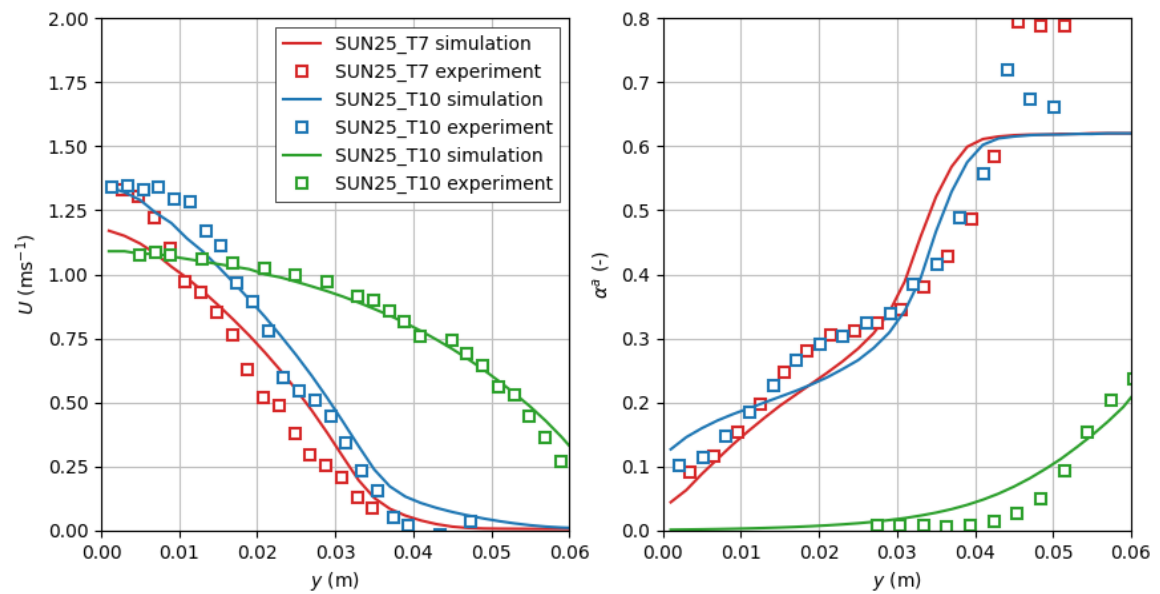

Figure 3: Velocity and particle concentration profiles optimized for the best agreement in $\alpha^{a}$ and $U$.

\section{$5.2 \quad 2-\mathrm{D}$ simulations}

In order to test the second hypothesis stated in the previous section, the 2-D simulations, corresponding to the experimental conditions of $\mathrm{T} 5, \mathrm{~T} 7$ and $\mathrm{T} 10$ cases, were conducted. In all the tested cases the steady-state profiles provided by the 2-D simulation differ from those of 1-D simulations. However, none of the simulated profiles provided a significantly better agreement between the experimental and simulated results. Moreover, an unexpected formation of periodic waves was observed on the interface between the stationary and moving bed. The waves with the frequency approx. $0.9-1.2 \mathrm{~Hz}$ and the amplitude of approx. $15 \mathrm{~mm}$ origin at the coordinate $x \approx 1.2 \mathrm{~m}$ and wash-out seriously the concentration and velocity profiles.

The illustration of the 2-D simulation results is shown in Fig. 4 as mean concentration profiles for $\mathrm{T} 7$ data set. The data are sampled at $x=4.0 \mathrm{~m}$ during the time period between $120 \mathrm{~s}$ and $140 \mathrm{~s}$. The error bars show the standard deviation of the averaged value. For the comparison, the results of the 1-D simulation are displayed. The origin of this phenomena was not yet explained. There is a possibility that the flow properties of the suspension (particle size, density, liquid viscosity and flow velocity) are prone to the natural instabilities like the Kelvin-Helmholz instability. This 


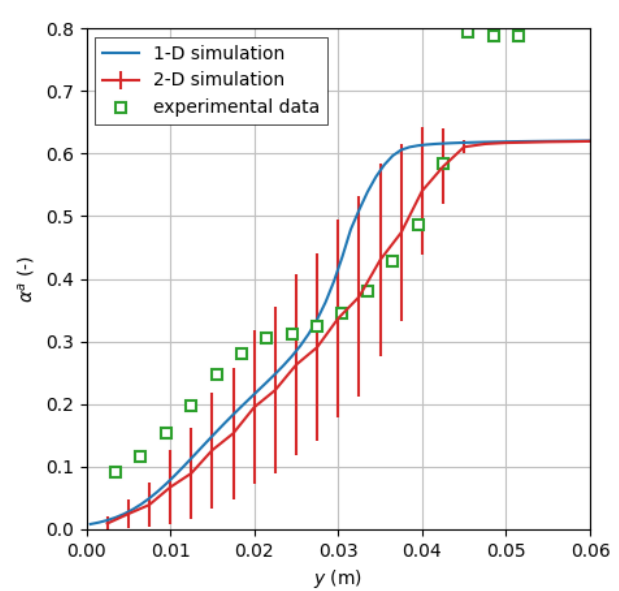

Figure 4: The results of 2-D simulation for T7 data set. The error-bars correspond to the standard deviations of the averaged values.

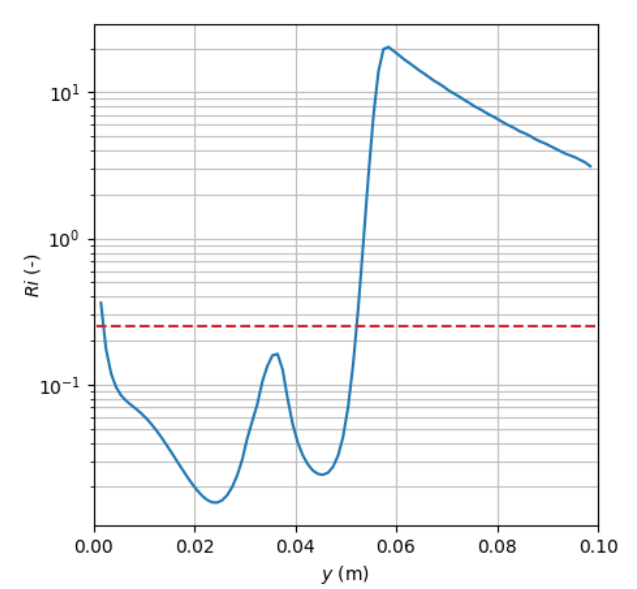

Figure 5: Distribution of the Richardson number along the channel height. The red line marks the critical value of 0.25 .

conclusion is supported by the distribution of the Richardson number,

$$
R i=\frac{g}{\rho} \frac{\partial \rho / \partial y}{(\partial U / \partial y)^{2}} \approx \frac{g}{\rho} \frac{\Delta \rho \Delta y}{(\Delta U)^{2}}
$$

along the channel height, which value drops bellow the critical 0.25 in the vicinity of stationary bed level - see Fig. 5. However, no such bed height fluctuation was observed.

On the other hand, the observed instability in the simulation results can be at least partially attributed to the term $\tilde{p}_{f}^{a}$ given by Eq. (24) [11, 12]. This artificial term is introduced in the solid phase momentum equation (1) to prevent the particle concentration $\alpha^{a}$ exceeding the value of the maximum packing density. The term $\nabla \tilde{p}_{f}^{a}$ is highly non-linear, and in the concentration range $\alpha^{a} \gtrsim 0.62$ it is very sensitive to the $\nabla \alpha^{a}$ value. As a consequence, even a small perturbation in the $\alpha^{a}$ results in the steep increase of $\nabla \tilde{p}_{f}^{a}$ to the order of magnitude $10^{5} \mathrm{~Pa} \cdot \mathrm{m}^{-1}$ promoting the further growth of the instability.

It was observed, that the waves, generated at the solid-bed interface, can be greatly suppressed if the individual components of $\nabla \tilde{p}_{f}^{a}$ are limited to a certain maximal value $\left|\frac{\partial \tilde{p}_{f}^{a}}{\partial x}\right|_{\max }$, i.e.,

$$
\left|\frac{\partial \tilde{p}_{f}^{a}}{\partial x_{i}}\right|=\min \left\{\left|\frac{\partial \tilde{p}_{f}^{a}}{\partial x_{i}}\right|,\left|\frac{\partial \tilde{p}_{f}^{a}}{\partial x}\right|_{\max }\right\}
$$

The value of the limiting value, however, must be carefully tuned because for the cases with the limit set too high, there was no or only negligible wave suppression, while for the too-low limit the $\nabla \tilde{p}_{f}^{a}$ is not large enough to effectively prevent the stationary bed particle concentration above the natural limit. The latter case results either in the further computational instabilities or in the mass and momentum unbalance. The simulated bed behavior for three degrees of $\nabla \tilde{p}_{f}^{a}$ limitations are shown in Fig. 6.

\section{Conclusions}

A series of 1-D and 2-D simulations is conducted in order to test the ability of the recent kinetic theory of granular flows to predict the characteristics of granular flow under turbulent conditions.

The results obtained in this work show the unsatisfactory ability of the kinetic theory to provide the concentration and velocity profiles in an acceptable agreement with the experimental data. 
no-limit

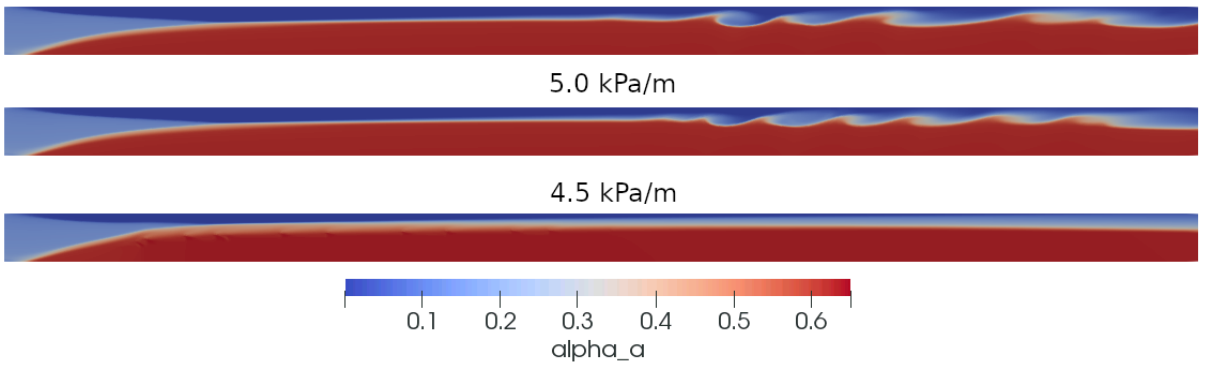

Figure 6: The illustration of simulation sensitivity to $\left|\partial \tilde{p}_{f}^{a} / \partial x\right|_{\max }$ value.

Moreover, a significant numerical instability was observed in the case of 2-D simulations, further increasing the computational expenses for such simulation and decreasing the simulation reliability.

In future research, the relations of the kinetic theory have to be modified to achieve better agreement between the experimental and simulation results. Especially the apparent need for significantly higher than experimentally utilized phase flow rates should be addressed. Alongside the KT improvement, the arbitrary term $\nabla \tilde{p}_{f}^{a}$ and its treatment in the sedFoam solver should be revised in order to stabilize the simulation and prevent the non-physical wave formation at the stationary-bed boundary.

\section{Acknowledgment}

This work was supported by Czech Science Foundation Grant No. 19-18411S and by the institutional support of the Czech Academy of Sciences [RVO: 67985874].

\section{References}

[1] Bonamy, C., Chauchat, J., Puig-Montella, E., Mathieu, A., Higuera, P. \& Hsu, T.-J.: Sedfoam/sedfoam: Release 3.1. (2020).

[2] The openfoam foundation: (Dec 2021). URL https://openfoam.org/.

[3] Chauchat, J., Cheng, Z., Nagel, T., Bonamy, C. \& Hsu, T.-J.: Sedfoam-2.0: a 3-d two-phase flow numerical model for sediment transport. Geoscientific Model Development. vol. 10 no. 12: (2017). pp. $4367-4392$.

[4] Gidaspow, D. Multiphase Flow and Fluidization. Academic Press: San Diego: (1994). ISBN 978-008-051226-6.

[5] Hsu, T.-J., Jenkins, J. T. \& Liu, P. L.-F.: On two-phase sediment transport: sheet flow of massive particles. Proceedings of the Royal Society of London. Series A: Mathematical, Physical and Engineering Sciences. vol. 460 no. 2048: (2004). pp. 2223-2250.

[6] Garzó, V. \& Dufty, J. W.: Dense fluid transport for inelastic hard spheres. Phys. Rev. E. vol. 59: (1999). pp. 5895-5911.

7] Berzi, D. \& Vescovi, D.: Different singularities in the functions of extended kinetic theory at the origin of the yield stress in granular flows. Physics of Fluids. vol. 27 no. 1: (2015). page 013302.

[8] Matoušek, V., Zrostlík, Š., Fraccarollo, L., Prati, A. \& Larcher, M.: Internal structure of intense collisional bedload transport. Earth Surface Processes and Landforms. vol. 44 no. 11: (2019). pp. 22852296.

[9] Capart, H., Young, D. L. \& Zech, Y.: Voronoï imaging methods for the measurement of granular flows. Experiments in Fluids. vol. 32 no. 1: (January 2002). pp. 121-135.

[10] Cheng, Z., Hsu, T.-J. \& Chauchat, J.: An eulerian two-phase model for steady sheet flow using large-eddy simulation methodology. Advances in Water Resources. vol. 111: (2018). pp. 205-223.

[11] Johnson, P. C. \& Jackson, R.: Frictional-collisional constitutive relations for granular materials, with application to plane shearing. Journal of Fluid Mechanics. vol. 176: (1987). page 67-93.

[12] Cheng, Z., Hsu, T.-J. \& Calantoni, J.: Sedfoam: A multi-dimensional eulerian two-phase model for sediment transport and its application to momentary bed failure. Coastal Engineering. vol. 119: (2017). pp. 32-50. 